\title{
PERSONAL GENOMICS APPROACH THROUGH NEXT GENERATION SEQUENCING: TOOL FOR PRECISION MEDICINES IN ENHANCING HUMAN HEALTH CARE
}

\author{
Yazhini P \\ B.Tech (Biotechnology) student, \\ Institute of Fisheries Biotechnology, \\ IFPGS Campus, Tamil Nadu Dr.J.Jayalalithaa Fisheries \\ University, OMR Campus, Vaniyanchavadi \\ Chennai-603103, Tamil Nadu, India
}

\author{
Dr. Suresh, E, M.F.Sc, Ph.D. \\ Assistant Professor \& Head, Department of FGB \\ Institute of Fisheries Post Graduate Studies (IFPGS) \\ Tamil Nadu Dr.J.Jayalalithaa Fisheries University, \\ OMR Campus, Vaniyanchavadi \\ Chennai-603103, Tamil Nadu, India
}

\author{
Dr. Kathirvelpandian, A, M.F.Sc, Ph.D. \\ Associate Professor \& Head, Department of Fisheries Biotechnology, \\ Institute of Fisheries Post Graduate Studies (IFPGS) \\ Tamil Nadu Dr.J.Jayalalithaa Fisheries University, \\ OMR Campus, Vaniyanchavadi \\ Chennai-603103, Tamil Nadu, India
}

\begin{abstract}
The advent of next-generation DNA sequencing technologies drastically brought down the cost of sequencing the human genome at reduced time. The speed and cost-effective sequencing of the human genome enables the application of genomic information in various aspects of disease prediction, diagnostics, and treatment of human diseases in an effective way. Despite, the limitations in the appropriate extraction of huge genome data and policy issues in the availability of the genome information of individuals, the personal genome plays an important role in customized treatment for human diseases such as cancer, etc., Personal genomics is the concept of understanding the information of individual's genome for a particular perspective. Personalized medicine utilizing the personal genomics approach is rapidly increasing in the field of health care which is generally based on the aspects of genetic and specific environmental conditions. Personalized medicine uses a molecular understanding of disease conditions specific to an individual in formulating preventive health care strategies and to begin drug therapies at the initial stages of disease itself. Thus, personal genomics is of immense use in medical care at an individual level resulting in exceptional customization of health care.
\end{abstract}

Keywords-Personal genome data, Next-Generation Sequencing, Precision Medicines, Human Health Care

\section{INTRODUCTION:}

Personal genomics is a branch of genomics that deals with generating genome information through sequencing, analysing using bioinformatic tools, and interpreting for a person. "Genomics and Medicine" is an emerging medical discipline and it is a part of customized individual clinical care (eg. diagnostics or therapeutics). Personal genomics could be effectively combined with social networking and genetics study. It involves personalized medicine, which is a part of health care based on the individual characteristics of each patient through understanding the unique molecular and genetic profile of individuals makes them predisposed susceptibility to certain diseases. Physicians can select a treatment or therapy protocol based on the patient's genomic profile (chen et al., 2011). Customized medicine through personal genomics marks an impact on pharmacology, pharmacokinetics in human diseases such as cancer, etc., Next Generation Sequencing (NGS) provides a notable step forward in personalized medicine by enabling the detection of somatic and germline mutation, which serve as the base for advanced precision-based human health care. The personalized genomics can facilitate the timely detection of cellular changes, degree of progression, and scope of survival (Willard et al., 2011). The present article includes the scope and advantages of personal genomics, tools used and future perspectives, etc., 


\section{International Journal of Engineering Applied Sciences and Technology, 2020 Vol. 4, Issue 12, ISSN No. 2455-2143, Pages 183-187 \\ Published Online April 2020 in IJEAST (http://www.ijeast.com)}

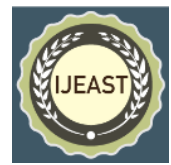

\section{ADVANTAGES OF PERSONAL GENOMICS:}

- Earlier disease intervention and management.

- Enables to apply more advanced medical decisions.

- Personalized medicine focuses majorly on general wellness and disease prevention.

- Highly specific diagnosis through genetic variations over other general symptoms.

- Reduction of probability of negative side-effects.

- Pharmacogenomics offers the opportunity to avoid severe toxicity in patients unable to adequately metabolize cytotoxic or potentially to adjust the drug dose to improve efficacy or to reduce toxicity.

- Prescribe more effective drugs.

\section{TOOLS IN PERSONAL GENOMICS:}

Bioinformatics tools play an important role in genomics as they are used in understanding the information useful for various diverse applications. Bioinformatics is a computational analysis of large and complex datasets of biological information using various tools that facilitates an enhanced discovery of drug targets and individual therapy. Genomic information can provide major contributions to drug therapy through identifying genes that coded for secreted proteins which will be the most suitable targets for drug intervention and to understand the response of the patients to various drugs and dosage. It involves in the utilization of information from the Human Genome Project and other related programs for identifying an increased number of targeted drugs. Some basic databases for genomic research are Genomic databases such as The Human Gene Mutation Database (HGMD) which consist a collection of mutations in the genes which are associated with human inherited diseases, Single nucleotide polymorphism (SNP) databases such as dbSNP, expression databases, etc., Bioinformatics software platforms such as MOSAIK, PANTHER,SUSPECT, ENDEAVOR, GeneMANIA, transMART, G-DOC are used in precision medicine using genome information (Teufel et al.,2006).

\section{ROLE OF SNPs IN PERSONAL GENOMICS:}

SNPs (Single Nucleotide Polymorphism) are molecular markers with single nucleotide change in the genome varies between individuals. The SNPs are effectively used to assess disease risk, carrier status, and drug response/interaction. Pharmacogenomics is improving the drug discovery process by accelerating target identification, thereby selecting the most promising drug candidates. Single Nucleotide polymorphism could be effectively used as a marker in understanding diseases such as Sickle cell anemia. SNPs are more frequently observed in persons with diseases than in non-affected ones.
Genetic variation in the human genome is an emerging resource for an understanding a complex set of diseases characterized by both environmental and genetic contributions. Genetic variation studies can bring out critical determinants under environmental exposure and diseases, which could have a direct implication for predictive and early intervention strategies. SNPs are used to rigorously analyze the genetic contributions to complex diseases, such as cancer. Before the process of generating a draft Human genome sequence, it has become clear that the extent of genetic variation is much larger than previously estimated. The draft human genome estimated more than 10 million SNPs with micro allele frequency over $10 \%$ is estimated to be perhaps as many as five million. SNP are distributed throughout the human genome, at a frequency of at least every 1000 base pair. The complexity of analyzing SNPs in a gene or locus can be reduced by the analysis of markers inherited in an individual inferred by Linkage Disequilibrium analysis of a region in unrelated subjects or through molecular characterization in family pedigrees (Erichsen, \& Chanock, 2004).

Most of the published articles examined at most few SNPs or genes, and on rare occasion variants within genes of a pathway or related biological process such as DNA repair enzymes (XRCC1 and XRCC3) or xenobiotic metabolism genes (NAT1 \& NAT2). In recent times, technical and bioinformatics advances made it possible to dramatically increase the number of SNPs linked with various types of cancer. It is important to bear in mind that SNP analysis requires replication before acceptance, and certainly before clinical implementation. The SNPs closely linked with the genes associated with various types of cancer would be studied in personal genomics data for early intervention through precision therapy.

\section{HUMAN GENOME PROJECT (HGP) AND RELEVANCE IN PERSONALIZED GENOMICS:}

The Human genome is made up of approximately three billion base pairs of deoxyribonucleic acid (DNA). The Human Genome Project (HGP) which was carried out from 1990 to 2003, provided researchers with basic information about the sequences of the three billion nucleotide base pairs. The HGP was further helped to improve the technologies needed to interpret and analyze genomic sequences, by identifying most of the genes encoded in the human genome.

The first draft sequence took 13 years to accomplish, not much more than a decade later, whole genome or exome sequencing using NGS platforms are becoming common in many hospital laboratories. These technological advances paved way to the saga of personalized medicine which utilizes the person's genetic profile to guide decisions to prevent, diagnosis, and treatment of disease effectively. This is a profound shift in addressing rare disorders with the use of genetic aspects of health care (Gonzalez and Manual, 2014). 


\section{International Journal of Engineering Applied Sciences and Technology, 2020 \\ Vol. 4, Issue 12, ISSN No. 2455-2143, Pages 183-187 \\ Published Online April 2020 in IJEAST (http://www.ijeast.com)}

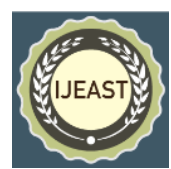

\section{GENOMIC INFORMATION TO PERSONALIZED MEDICINE:}

Personalized medicine is conceived as more broadly applicable across health care as it includes the strategy of genomic profiling to identify the individual risk factors for multifactorial diseases (eg. Cardiovascular disease, cancer and type 2 diabetes) where disease risk results from the interaction between several genes (polygenic) as well as non-genomic factors.

Genomic profiling often involves measuring the variation in SNPs associated with the diseases. While SNPs may lead to a mutation that causes monogenic disorder (which are generally rare, usually to cause disease which are more readily identifiable as causative). Integration of genomic testing with other clinical investigations, delivered by health care professionals is termed as Genomic in medicine. Screening of disease in personalized medicine takes a major role in diagnosing the disease early through identifying various gene mutations (Wilson et al., 2015).

\section{PERSONAL GENOMICS IN MEDICAL FIELD:}

Personalized medicine has a transformative impact on personal health, health economics and productivity. In recent times, there has been an increased leap in the knowledge of the human genome and their role in human health. Currently, genome sequencing having the greatest impact in stratifying cancer, characterizing genetic disorders and providing information about an individual's likely response to treatment.

The fundamental premise of cancer genomics is that cancer is caused by somatically acquired mutations in the genome of an individual. Despite, capillary-based cancer sequencing has been ongoing for over a decade; these investigations were restricted to a few samples and candidate genes. With the advent of NGS, cancer genomes can now be systematically studied fully. This might provide many benefits including a more precise diagnosis and classification of the disease, accurate prognosis and the identification of 'drug-able' casual mutation.

Individual genome sequencing might provide the basis of personalized cancer management. NGS of cancer genomics in clinical practice, mainly aiming to identify mutations in tumours that can be targeted by mutation-specific drugs (Mattick et al., 2014).

\section{Classifying tumours for treatment}

Genomic medicine has already shown benefit in refining diagnoses and guiding the therapeutic approaches for cancer treatment. In recent times, cancer treatment is supplemented by therapies that target specific molecular pathways in cancer growth and development. In genomic medicine, the clinician can decide treatment strategies by classifying a tumour according to its mutation and corresponding drug sensitivities. Clinicians are using genomic information to refine a cancer diagnosis, prognosis, and treatment.

Genome - wide sequencing (WGS) is applied to analyze circulating DNA in the plasma of patients of cancer and other diseases. This technology enables non-invasive detection of cancer and checking the response to therapy that promise to significantly improve patient management.

\section{Characterizing and diagnosing of diseases:}

Genome sequencing is increasingly used to assess genetic aspects to complex diseases, where multiple gene variants are involved in disease development and progression.

Despite sequencing the entire genome, rapid sequencing of the protein - coding portion can provide a comparatively costeffective analysis of many genetic disorders. Although less effective in detecting chromosomal duplications, deletions, and rearrangements, exome sequencing identifies most clinically relevant nucleotide variants in a single analysis. WGS is likely to provide a clinically more robust, accurate, and useful that will replace exome sequencing.

\section{Linking molecular and clinical information:}

The pathway from the patient's genome sequencing to a medical treatment plan relies on the linking of the individual's genomic information with knowledge databases that contain genomic and clinical associations from large populations of individuals.

The inherent computational challenges in integrating genomic and clinical data need a significant investment in bioinformatics.

\section{Drug and dosage prescription:}

Cancer treatment is benefited from the genomic information to predict how an individual will respond to drugs (pharmacogenomics) and to ascertain an appropriate drug or dosage. 


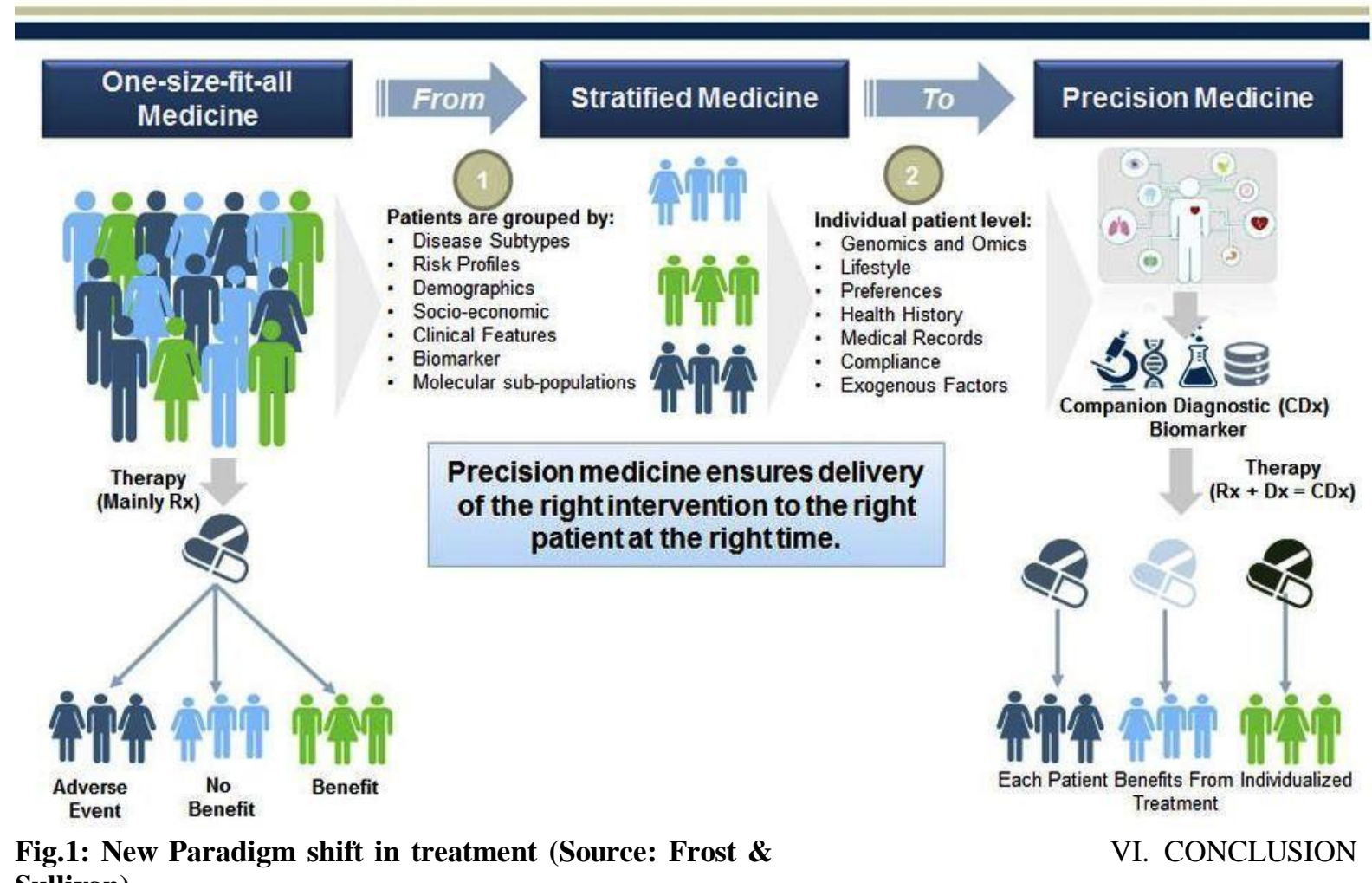

Sullivan)

\section{ETHICAL ISSUES}

Genomic studies require human genetic material, from a large number of individuals for analyzing accurate personal genome analysis for precision medicine. New types of ethical, legal, and social issues (ELSI) have arisen due to the genomic analysis of the large human populations. The ELSI program was founded in 1990 as an integral part of the Human Genome Programme.

ELSI program was aimed to identify and address issues raised by genomic research that would affect individuals and society. The ELSI program focused on the possible consequences of genomic research in four main areas:

- Privacy in the use of genetic information.

- Integration of genetic testing into the practice of clinical medicine.

- Ethical issues surrounding the design and conduct of genetic research for carrying out the research with informed consent.

- Educating healthcare professionals, policymakers, and the public about complex issues resulting from genomic research.

Personal genome is a powerful utility of genetic information in human health management. It needs to access sequence data and analyze the genomes of considerable people across the world. Further, the commercialization of personal genome sequencing is set to advance in the future, could become a routine in clinical practices. It involves in various fields of human health in diagnosing, preventing and treating various genetic disorders. Personal Genomics is also used to check the carrier of genetic disorders. By carefully managing societal and human ethics issues, personal genomics and personalized medicine could be useful significantly in human health.

\section{REFERENCE}

[1] AL-Dewik, Nader I, and M. Walid Qoronfleh (2019)."Genomics and Precision Medicine: Molecular Diagnostics Innovations Shaping the Future of Healthcare in Qatar". Advances in Public Health. doi.org/10.1155/2019/3807032 (11 pages).

[2] Chan, Isaac S., and Geoffrey S. Ginsburg (2011) "Personalized medicine: progress and promise". Annual review of genomics and human genetics 12: 217-244.

[3] Christoph Bock. New tools for personalized medicine-first research workshop, Milano, 26 June 2017. 


\section{International Journal of Engineering Applied Sciences and Technology, 2020 \\ Vol. 4, Issue 12, ISSN No. 2455-2143, Pages 183-187 \\ Published Online April 2020 in IJEAST (http://www.ijeast.com)}

[4] Ellsworth, Rachel, David J Decewicz, Craig D Shriver, and Darrell L Ellsworth (2010). "Breast cancer in the personal genomics era." Current genomics 11, no. 3: 146-161.

[5] Erichsen, H. C., and Chanock, S. J. (2004). "SNPs in cancer research and treatment". British journal of cancer, 90(4), 747-751.

[6] Ginsburg, Geoffrey S., and Huntington F. Willard (2008). Genomic and personalized medicine. Vol. 1. Academic Press,

[7] Ginsburg, Geoffrey S., and Huntington F. Willard. "Genomic and personalized medicine: foundations and applications." Translational research 154, no. 6 (2009): 277-287.

[8] Gonzalez-Garay, Manuel L (2014). "The road from next-generation sequencing to personalized medicine." Personalized medicine 11, no. 5: 523-544.

[9] Human Genome Project.,https://en.m.wikipedia.org

[10] Jinek, Martin, Krzyszt of Chylinski, Ines Fonfara, Michael Hauer, Jennifer A. Doudna, and Emmanuelle Charpentier (2012). "A programmable dual-RNAguided DNA endonuclease in adaptive bacterial immunity." Science 337, no. 6096: 816-821.

[11] Kato, K., and J. Minari (2013). "Ethical issues in personal genome research." Brain and nerve= Shinkei kenkyu no shinpo 65, no. 3: 267-272

[12] Lunshof, Jeantine E., Jason Bobe, John Aach, Misha Angrist, Joseph V. Thakuria, Daniel B. Vorhaus, Margret R. Hoehe, and George M. Church (2010). "Personal genomes in progress: from the human genome project to the personal genome project." Dialogues in clinical neuroscience 12, no. 1: 47

[13] Mattick, John S., Marie A. Dziadek, Bronwyn N. Terrill, Warren Kaplan, Allan D. Spigelman, Frank G. Bowling, and Marcel E. Dinger (2014). "The impact of genomics on the future of medicine and health." Medical Journal of Australia 201, no. 1: 1720

[14] Personal genomics,https://innovatemedtec.com

[15] Personal genomics,wikipedia "nci dictionary of cancer terms" nci $2^{\text {nd }}$ feb, 2011

[16] Sanger sequencing,en.m.wikipedia.org

[17]Tafe, Laura J (2019). "Genomic Medicine: A Practical Guide”. Springer Nature.

[18] Teufel, Andreas, Markus Krupp, Arndt Weinmann, and Peter R. Galle (2006). "Current bioinformatics tools in genomic biomedical research." International journal of molecular medicine" 17, no. 6: 967-973.

[19] Vogenberg, F. Randy, Carol Isaacson Barash, and Michael Pursel (2010). "Personalized medicine: part 1: evolution and development into theranostics." Pharmacy and Therapeutics" 35, no. 10: 560
[20] Willard HF, Ginsburg Personalized (2009). Amsterdam: Elsevier. 1478 pp.

[21] Wilson, Brenda J., and Stuart G. Nicholls (2015). "The Human Genome Project, and recent advances in personalized genomics." Risk management and healthcare policy" 8: 9. 